\title{
Solvent free Lewis acid catalyzed vinylogous condensation
}

\author{
Gitalee Bhattacharjya, Sarit. S. Agasti, and Gurunath Ramanathan* \\ Department of Chemistry, Indian Institute of Technology, Kanpur - 208 016, India \\ E-mail:gurunath@iitk.ac.in
}

\begin{abstract}
(4Z)-4-(4-Methoxybenzylidene)-2-((E)-4-methoxystyryl)-1-phenyl-1,4-dihydro-5H-imidazolin5 -one and its analogues were synthesized via carbon-carbon bond formation in the presence of a variety of Lewis acids under essentially solvent free conditions. $\mathrm{ZnCl}_{2}$ was used as the prototype catalyst in these studies. Several other Lewis acids were also tested in this synthesis with $\mathrm{AlCl}_{3}$ giving very good yields. Protic acids also catalyzed the reaction albeit at much higher mole percents. All 2-methyl substituted imidazolin-5-one starting materials resulted in the formation of the $\pi$-conjugated product on reaction with aromatic aldehydes. The mechanism of this reaction has also been elucidated.
\end{abstract}

Keywords: Vinylogous condensation, Lewis acid, catalyst, solvent-free, imidazolin-5-ones, $\pi$ conjugated systems

\section{Introduction}

Condensation reactions are used in organic synthesis and biochemical systems as a synthetic route leading to new carbon-carbon bonds. ${ }^{1,2}$ In this context, the vinylogous reactions play an important part. $^{3}$ More vividly, among vinylogous reactions, the aldol addition reaction has attracted considerable interest over the years as a popular tool for the construction of new carbon-carbon bonds. ${ }^{4,5,6}$ In this class of reactions, the best studied reaction involves the addition of silyl enol ethers to carbonyl compounds in the presence of Lewis bases ${ }^{7}$ and Lewis acids ${ }^{8}$ as catalyst (Mukaiyama aldol condensation). ${ }^{4,9}$ A few other aldol condensations catalyzed by Brönsted bases as well as Lewis acids, to form a metal enolate in situ have also been reported recently in the literature. ${ }^{10}$ Several attempts have resulted in the successful enhancement of the yields of the reaction by changing reaction conditions (such as aqueous medium). ${ }^{5,11}$

An active methyl or methylene group on aldehydes appears to be an essential precursor for these reactions to proceed. ${ }^{12,13}$ In this, the initial step is the formation of a carbanion, by the action of a base. It is then followed by condensation in the presence of a Brönsted base or Lewis acid to form the new carbon-carbon bond, i.e. the condensation product. ${ }^{13}$ Direct condensation in 
the presence of a Lewis acid or base has also been observed over the years. ${ }^{5}$ But in this context, the results were appreciable only in the presence of a highly acidic methyl or methylene group which will initiate the condensation reaction via carbanion formation. ${ }^{5}$

Organic reactions under solvent-free ${ }^{14}$ conditions have also increasingly attracted chemist's interest particularly from the viewpoint of green chemistry. We report here the isolation of a series of imdazolin-5-ones (Scheme 1) with a $\pi$-conjugated system from (4Z)-4-(4methoxybenzylidene)-2-methyl-1-phenyl-1,4-dihydro-5H-imidazolin-5-one $\mathbf{1}^{15,16}$ and a variety of aromatic aldehydes in the presence of Lewis acid catalysts under solvent free conditions. (4Z)4-(4-methoxybenzylidene)-2-((E)-4-methoxystyryl)-1-phenyl-1,4-dihydro-5H-imidazolin-5-one 2a was the first compound to be synthesized in this series.

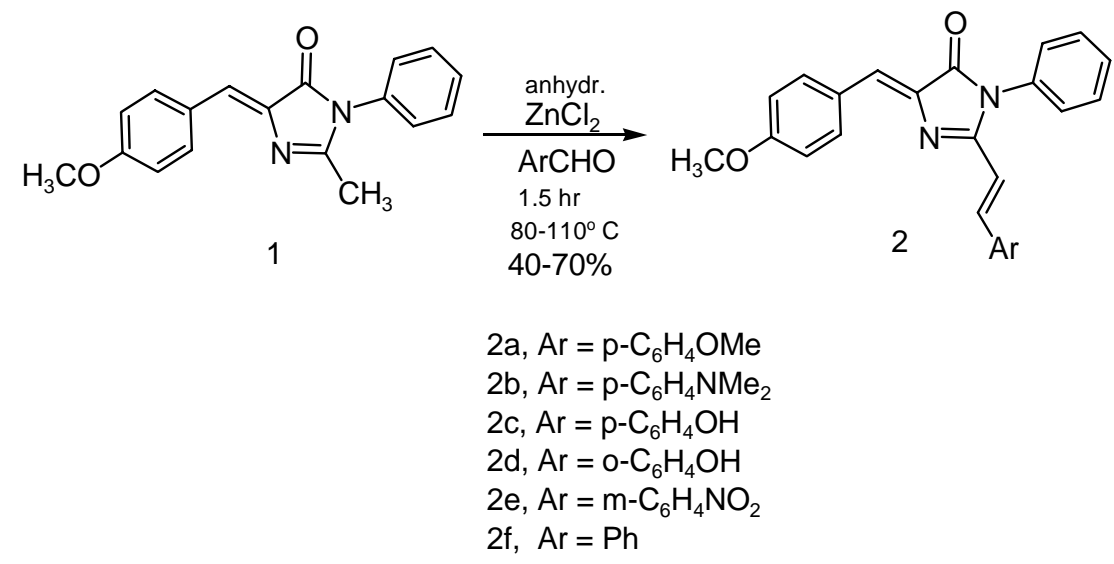

\section{Scheme 1}

\section{Results and Discussion}

The starting material 1 for this reaction was synthesized from its corresponding oxazolone following the reported procedure. ${ }^{15,17}$ When the imidazolin-5-one $\mathbf{1}$ was reacted with $p$ anisaldehyde in a fusion reaction in the presence of a mild Lewis acid $\mathrm{ZnCl}_{2}$, work up and column chromatography of the reaction mixture yielded an orange product in $50-55 \%$ yield, which was characterized as (4Z)-4-(4-methoxybenzylidene)-2-((E)-4-methoxystyryl)-1-phenyl1,4-dihydro-5H-imidazolin-5-one 2 a.

The reaction was carried out under anhydrous conditions. The reason for this is that the presence of even a small amount of water hinders the reaction as most Lewis acids react immediately with water rather than the substrates, and decompose or deactivate. ${ }^{13}$

The mechanism of this reaction is proposed to be a Lewis acid catalyzed vinylogous condensation. A methyl substituent is weakly acidic to initiate the condensation reaction in the absence of a base. But in compound 1, the orientation of bonds facilitates the delocalization of electrons within the benzylidene-imidazolinone ring system, making the methyl substituent 
more acidic. This delocalization of electrons provides a partial double bond character to the methyl substituent, thereby creating the stage for the initiation of the reaction. The Lewis acid first activates the non-substituted nitrogen atom in the imidazolinone ring and then activates the aldehyde via the formation of a six-membered cyclic transition state. This in turn facilitates the formation of a new carbon-carbon double bond with the elimination of water. The mechanism of this typical reaction is shown in Scheme 2.

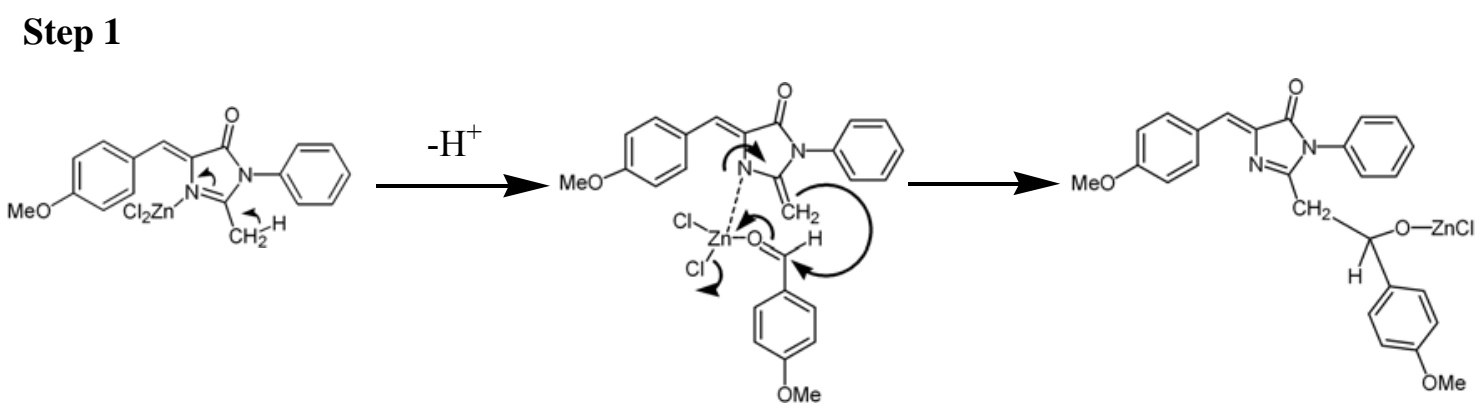

Step 2

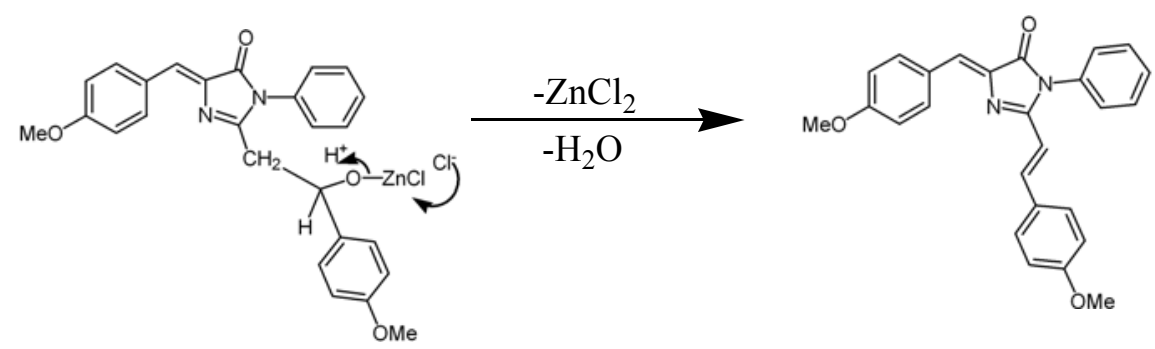

\section{Scheme 2}

The prediction of the formation of a double bond character of the methyl substituent was further verified by deuterium exchange on 1. ${ }^{1} \mathrm{H}$ NMR $(400 \mathrm{MHz})$ studies on 1 in a mixture of $\mathrm{CDCl}_{3}$ and $\mathrm{D}_{2} \mathrm{O}$ showed a loss of proton from the methyl substituent as measured by the integration values (figure 1). An upfield chemical shift was also observed on deuterium exchange on 1. This together with the $\mathrm{C}=\mathrm{O} \bullet \cdot \mathrm{C}$ interaction previously reported by $\mathrm{us}^{16}$ proved that the methyl substituent is acidic enough to initiate this reaction.

This vinylogous condensation reaction was tried with various other starting materials such as (4Z)-4-(4-bromobenzylidene)-2-methyl-1-phenyl-1,4-dihydro-5H-imidazolin-5-one, (4Z)-4(2-methoxybenzylidene)-2-methyl-1-phenyl-1,4-dihydro-5H-imidazolin-5-one,

$(4 Z)-4-(2-$ chlorobenzylidene)-2-methyl-1-phenyl-1,4-dihydro-5H-imidazolin-5-one, (4Z)-4-(4cyanobenzylidene)-2-methyl-1-phenyl-1,4-dihydro-5H-imidazolin-5-one and (4Z)-4benzylidene-2-methyl-1-phenyl-1,4-dihydro-5H-imidazolin-5-one. All of these starting materials showed the formation of the product with different aldehydes and under Lewis acid catalyzed conditions. The reaction was further probed under different Lewis acid catalyzed conditions. In 
compound 1, the methoxy group in the benzylidene ring, in the para position acts as a good electron donor. This enables the delocalization of electrons throughout the molecule in the correct way to initiate the reaction, thereby, proving to be a suitable substrate to undergo further condensation reactions with an appreciable yield. On the contrary, electron withdrawing groups such as a cyano group instead of a methoxy group also allowed the reaction to proceed under the same reaction conditions, but with very low yields (15-20\%).

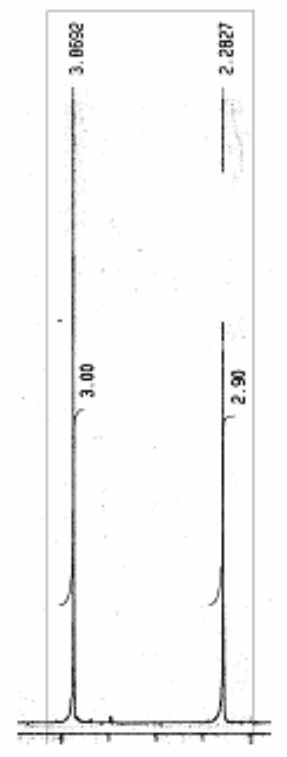

Before deuterium addition

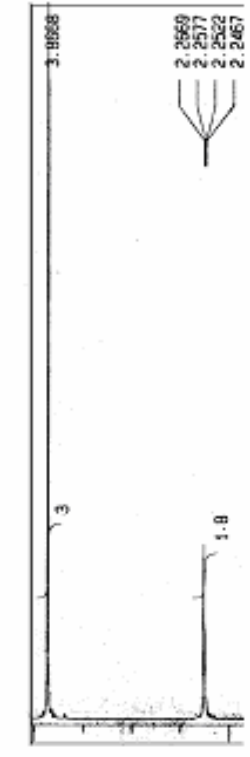

After 24 h of deuterium addition

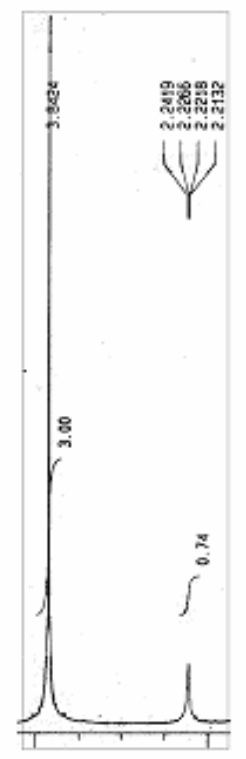

After 5 days of deuterium addition

Figure 1. ${ }^{1} \mathrm{H}$ NMR spectra of compound 1 before and after deuterium exchange. The ratio of the integration value of methyl protons before deuterium exchange is 2.90 and after $24 \mathrm{~h}$ of deuterium exchange is 1.8 indicating the loss of a proton on deuterium exchange. After 5 days two protons get exchanged and eventually with time all the protons get exchanged.

We have studied the reaction of compound 1 and various other aldehydes using $\mathrm{ZnCl}_{2}$ as catalyst at a temperature range between $80-110^{\circ} \mathrm{C}$ (scheme 1). The results are summarized in Table 1.

From Table 1 it can be concluded that the reaction of compound 1 with $p$-anisaldehyde and $p$-dimethylaminobenzaldehyde yielded product $\mathbf{2 a}$ and $\mathbf{2 b}$ with a yield of $55 \%$. The yield of compound $\mathbf{2 c}$ seems to be less than $\mathbf{2 a}$ and $\mathbf{2 b}$ due to the less electron donating character of $p$ hydroxybenzaldehyde than $p$-anisaldehyde and $p$-dimethylaminobenzaldehyde. Further, due to steric hindrance in $o$-hydroxybenzaldehyde, the yield of $2 \mathbf{d}$ was reduced to $45 \%$. In case of compound 2e, the presence of an electron-withdrawing nitro group has also reduced its yield to $45 \%$. On the contrary, benzaldehyde proved to be promising yielding compound $2 \mathbf{f}$ with a higher 
yield of $70 \%$. The absence of any substituent in benzaldehyde acts as a driving force for the stabilization of the $\pi$-conjugated system, thereby forming compound $\mathbf{2} \mathbf{f}$ in appreciable yield.

In our study reported here, we have tried different reaction conditions (Table 2), with $\mathrm{ZnCl}_{2}$ as Lewis acid and observed similar product yields.

Table 1. Comparative study of $\mathbf{1}$ with different aldehydes

\begin{tabular}{cccc}
\hline S. No & Aldehyde Used & $\begin{array}{c}\text { Isolated yield of } \\
\text { Product }^{\mathrm{a}}\end{array}$ & $\mathrm{m} . \mathrm{p}\left({ }^{\circ} \mathrm{C}\right)$ \\
\hline 1 & $55 \%$ & $123-124$ \\
\hline
\end{tabular}

${ }^{\text {a }}$ Reaction performed 3-5 times (average yield shown)

Table 2. Comparative study under different reaction conditions with $\mathrm{ZnCl}_{2}$ as Lewis acid

\begin{tabular}{ccccc}
\hline S. No & $\begin{array}{c}\text { Lewis acids } \\
\text { used }\end{array}$ & Conditions & $\begin{array}{c}\text { Amount used in } \\
\text { Mole } \%\end{array}$ & $\begin{array}{c}\text { Isolated Product Yield } \\
(\%)^{\mathrm{a}}\end{array}$ \\
\hline $\mathbf{1}$ & $\mathrm{ZnCl}_{2}$ & Solvents* $(2-3 \mathrm{~h})$ & 10 & 55 \\
$\mathbf{2}$ & $\mathrm{ZnCl}_{2}$ & $\begin{array}{c}\text { Microwave }(15-20 \\
\text { min) }\end{array}$ & 10 & 55 \\
3 & $\mathrm{ZnCl}_{2}$ & $\begin{array}{c}\text { Without solvents } \\
(1.5 \mathrm{~h})\end{array}$ & 10 & 55 \\
\hline
\end{tabular}

${ }^{\mathrm{a}}$ Reaction performed 3-5 times (average yield shown) 
We also tried other Lewis acids, both mild and strong ones compared to $\mathrm{ZnCl}_{2}$ and measured the yields under the same reaction conditions. For these studies, compound $\mathbf{1}$ and $p$ anisaldehyde were used, as the starting materials and the resultant product was compound 2a. The results are compiled in Table 3.

Table 3. Comparative study under different acidic condition

\begin{tabular}{cccc}
\hline S. No & Lewis acids used & $\begin{array}{c}\text { Amount used in mole } \\
\%\end{array}$ & $\begin{array}{c}\text { Isolated product yield } \\
(\%)^{\text {a }}\end{array}$ \\
\hline $\mathbf{1}$ & $\mathrm{ZnCl}_{2}$ & 1 & 75 \\
& & 10 & 55 \\
$\mathbf{2}$ & $\mathrm{MgCl}_{2}$ & 100 & 55 \\
$\mathbf{3}$ & $\mathrm{Cu}\left(\mathrm{OTf}_{2}\right.$ & 10 & 40 \\
$\mathbf{4}$ & $\mathrm{AlCl}_{3}$ & 10 & 35 \\
$\mathbf{5}$ & $\mathrm{BF}_{3} \cdot \mathrm{OEt}_{2}$ & 10 & 75 \\
$\mathbf{6}$ & $\mathrm{Conc} \mathrm{HCl}_{\mathbf{7}}$ & 10 & 55 \\
$\mathbf{8}$ & Acidic Alumina & 99 & 80 \\
$\mathbf{8}$ & Without acid/base & 10 & 55 \\
\hline
\end{tabular}

${ }^{a}$ Reaction performed 3-5 times (average yield shown)

It has been observed that in presence of milder Lewis acids with respect to zinc chloride such as anhydrous copper triflate and anhydrous magnesium chloride, product 2a was obtained with comparatively lower yield. With $\mathrm{BF}_{3}$. OEt as the Lewis acid catalyst, the yield has increased to $\sim 50 \%$, which is almost similar to $\mathrm{ZnCl}_{2}$. On the other hand with a strong Lewis acid such as aluminum chloride the yield of compound 2a was considerably increased to $75 \% . \mathrm{AlCl}_{3}$, being a strong Lewis acid, activates the $\mathrm{C}=\mathrm{O}$ group to a greater extend resulting in a promising yield of 2a. Further, protic acids also resulted in appreciable yields albeit at a higher mole percent of the catalyst. In the absence of acid or base, no product was formed. Moreover, lower mole $\%$ of the Lewis acid resulted in a much higher yield as observed from table 3.

\section{Conclusions}

In summary, the Lewis acid catalyzed vinylogous condensation of methyl substituted imidazolin5 -ones proves to be a highly effective method for the formation of $\pi$-conjugated heterocyclic systems. ${ }^{12}$ To the best of our knowledge, the synthesis of several of these compounds is being reported for the first time. Studies with different Lewis acids conclusively show that stronger Lewis acids like aluminum chloride provide excellent yields. We have also aimed at using these 
$\pi$ - conjugated products in organic semiconducting diodes ${ }^{18,19}$ which is a recent field of research interest.

\section{Experimental Section}

General Procedures. Melting points were determined on a JSGW apparatus. The purity of the compounds was checked by TLC (chloroform/petroleum ether, 4:1). The IR spectra were recorded on a BRUKER, VECTOR 22 FTIR system, $(0.5 \mathrm{~mm} \mathrm{KBr}$ cells at concentrations $(<0.5 \mathrm{mg} \mathrm{m} 21)$ ). ${ }^{1} \mathrm{H}$ NMR spectra were recorded on a Jeol LA-400 (400 MHz) NMR spectrometer in solution of $\mathrm{CDCl}_{3}$ using tetramethylsilane (TMS) as internal standard. The electrospray mass spectrum was recorded in CDRI Lucknow on a MICROMASS QUATTRO II triple quadrupole mass spectrometer. The sample (dissolved in methanol) was introduced into the ESI source through a syringe pump. The ESI capillary was set at $3.5 \mathrm{KV}$ and the core voltage was 40V. C,H,N \& O data analysis was provided by SAIF, CDRI Lucknow.

General procedure for the preparation of compounds 2a-f. (4Z)-4-(4-Methoxybenzylidene)2-methyl-1-phenyl-1,4-dihydro-5H-imidazolin-5-one 1 (0.1g, 0.34mmol), freshly distilled aromatic aldehyde $(0.05 \mathrm{~mL}, 0.41 \mathrm{mmol})$ and anhydrous zinc chloride $(0.005 \mathrm{~g}, 0.034 \mathrm{mmol})$ was fused at $80-110^{\circ} \mathrm{C}$ for 1.5 hours. The brown colored reaction mixture was extracted with EtOAc, after workup with water and then purified by column chromatography to yield an orange crystalline product with a solvent system of $10 \%$ ethyl acetate in petroleum ether.

(4Z)-4-(4-Methoxybenzylidene)-2-((E)-4-methoxystyryl)-1-phenyl-1,4-dihydro-5H-

imidazolin-5-one (2a). Isolated yield 55\%; m.p $123^{\circ}-124^{\circ} \mathrm{C} ; \mathrm{R}_{\mathrm{f}} 0.5\left(80 \% \mathrm{CHCl}_{3}\right.$-Petroleum ether); IR (KBr): 3433, 3069, 2922, 2853, 1707, 1627, 1600, 1512, 1373, 1259, 1169,1026 cm ${ }^{1} \mathrm{H}$ NMR (400 MHz, $\mathrm{CDCl}_{3}$ ): $\delta 3.74\left(\mathrm{~s}, 3 \mathrm{H}, \mathrm{OCH}_{3}\right), 3.80\left(\mathrm{~s}, 3 \mathrm{H}, \mathrm{OCH}_{3}\right), 6.37-6.40$ ( d, J=15.6 $\mathrm{Hz}, 1 \mathrm{H},=\mathrm{CHAr}), 6.78-6.80(\mathrm{~d}, J=8.04 \mathrm{~Hz}, 2 \mathrm{H}, \mathrm{ArH}), 6.92-6.93(\mathrm{~d}, J=8.28 \mathrm{~Hz}, 2 \mathrm{H}, \mathrm{ArH})$, $7.18(\mathrm{~s}, 1 \mathrm{H},=\mathrm{CHAr}), 7.24-7.26(\mathrm{~d}, J=7.88 \mathrm{~Hz}, 2 \mathrm{H}, \mathrm{ArH}), 7.34-7.47$ ( m, 5H, ArH), 7.88-7.92 $(\mathrm{d}, J=15.8 \mathrm{~Hz}, 1 \mathrm{H},=\mathrm{CHAr}), 8.18-8.20(\mathrm{~d}, J=8.28 \mathrm{~Hz}, 2 \mathrm{H}, \mathrm{ArH}) .{ }^{13} \mathrm{C} \mathrm{NMR}(400 \mathrm{MHz}$, $\left.\mathrm{CDCl}_{3}\right):$.55.31, 111.11, 114.31, 127.24, 127.50, 127.66, 128.55, 129.56, 133.45, 134.25, 136.97, 140.28, 157.92, 161.27, 169.99; $\operatorname{ESIMS~(m/z,~\% ):~411(M+1,~100).~Anal.~Calcd~for~}$ $\mathrm{C}_{26} \mathrm{H}_{22} \mathrm{~N}_{2} \mathrm{O}_{3}$ : C, 76.10; H, 5.41; N, 6.83; O, 11.70. Found: C, 76.11; H, 5.43; N, 6.93; O, 11.57

(4Z)-4-(4-Methoxybenzylidene)-2-((E)-4- $N$, $N$-dimethylaminostyryl)-1-phenyl-1,4-dihydro-

$5 \boldsymbol{H}$-imidazolin-5-one (2b). Orange product; Isolated yield 55\%; m.p $147^{\circ}-148^{\circ} \mathrm{C} ; \mathrm{R}_{\mathrm{f}} 0.5(80 \%$ $\mathrm{CHCl}_{3}$-Petroleum ether); IR (KBr): 3433, 3069, 2956, 2923, 2853, 2367, 1706, 1627, 1599 , 1526, 1504, 1362, 1304, 1257, 1162, $1029 \mathrm{~cm}^{-1}$; ${ }^{1} \mathrm{H}$ NMR $\left(400 \mathrm{MHz}, \mathrm{CDCl}_{3}\right): \delta 2.99(\mathrm{~s}, 6 \mathrm{H}$, $\left.\mathrm{CH}_{3}\right), 3.86\left(\mathrm{~s}, 3 \mathrm{H}, \mathrm{OCH}_{3}\right), 6.31-6.35(\mathrm{~d}, J=15.8 \mathrm{~Hz}, 1 \mathrm{H},=\mathrm{CHAr}), 6.60-6.63(\mathrm{~d}, J=8.8 \mathrm{~Hz}, 2 \mathrm{H}$, ArH), 6.97-6.99 (d, J=8.8 Hz, 2H, ArH), 7.24 (s, 1H, =CHAr), 7.30-7.32 (d, J=8.8 Hz, 2H, ArH), 7.35-7.53 ( m, 5H, ArH), 7.94-7.98 (d, $J=15.8 \mathrm{~Hz}, 1 \mathrm{H},=\mathrm{CHAr}), \quad 8.24-8.26(\mathrm{~d}, J=8.8$ $\mathrm{Hz}, 2 \mathrm{H}, \mathrm{ArH}) .{ }^{13} \mathrm{C} \mathrm{NMR}\left(400 \mathrm{MHz}, \mathrm{CDCl}_{3}\right): \delta$ 42.15, 55.31, 111.11, 114.31, 127.24, 127.50, 
$127.66,128.55,129.56,133.45,134.25,136.97,140.28,157.92,161.27,169.99 ;$ ESIMS ( $m / z$ $\%): 424(\mathrm{M}+1,100)$.

(4Z)-4-(4-Methoxybenzylidene)-2-((E)-4-hydroxystyryl)-1-phenyl-1,4-dihydro-5Himidazolin-5-one (2c). Orange product; Isolated yield 50\%; m.p $192^{\circ}-193^{\circ} \mathrm{C} ; \mathrm{R}_{\mathrm{f}} 0.5(80 \%$ $\mathrm{CHCl}_{3}$-Petroleum ether); IR (KBr): 3430, 3300, 3069, 2922, 2853, 1692, 1627, 1599, 1511, $1383,, 1257,1165,1028 \mathrm{~cm}^{-1} ;{ }^{1} \mathrm{H} \mathrm{NMR}\left(400 \mathrm{MHz}, \mathrm{CDCl}_{3}\right): \delta 3.89\left(\mathrm{~s}, 3 \mathrm{H}, \mathrm{OCH}_{3}\right), 5.14(\mathrm{~s}, 1 \mathrm{H}$, $\mathrm{OH}), 6.43-6.47$ ( d, $J=15.8 \mathrm{~Hz}, 1 \mathrm{H},=\mathrm{CHAr}), 6.80-6.82$ (d, $J=8.5 \mathrm{~Hz}, 2 \mathrm{H}, \mathrm{ArH}), 6.99-7.01$ (d, $J=8.8 \mathrm{~Hz}, 2 \mathrm{H}, \mathrm{ArH}), 7.20(\mathrm{~s}, 1 \mathrm{H},=\mathrm{CHAr}), 7.31-7.33$ (d, $J=8.4 \mathrm{~Hz}, 2 \mathrm{H}, \mathrm{ArH}), 7.38-7.54(\mathrm{~m}$, 5H, ArH), 7.94-7.98 (d, $J=15.8 \mathrm{~Hz}, 1 \mathrm{H},=\mathrm{CHAr}), 8.25-8.27(\mathrm{~d}, J=8.8 \mathrm{~Hz}, 2 \mathrm{H}, \mathrm{ArH}) .{ }^{13} \mathrm{C}$ NMR $\left(400 \mathrm{MHz}, \mathrm{CDCl}_{3}\right): \delta 55.44,111.11,114.31,116.19,119.01,124.46,127.50,127.66$, $128.55,129.56,130.41,133.45,134.25,136.97,140.28,157.92,161.27,174.11,207.25$; ESIMS $(\mathrm{m} / \mathrm{z}, \%): 397(\mathrm{M}+1,100)$.

(4Z)-4-(4-Methoxybenzylidene)-2-((E)-2-hydroxystyryl)-1-phenyl-1,4-dihydro-5H-

imidazolin-5-one (2d). Orange product; Isolated yield 45\%; m.p $142^{\circ}-143^{\circ} \mathrm{C} ; \mathrm{R}_{\mathrm{f}} 0.5(80 \%$ $\mathrm{CHCl}_{3}$-Petroleum ether); IR (KBr): 3420, 3345, 3069, 2920, 2853, 1692, 1627, 1599, 1511 , $1383,, 1257,1165,1028 \mathrm{~cm}^{-1} ;{ }^{1} \mathrm{H}$ NMR $\left(400 \mathrm{MHz}, \mathrm{CDCl}_{3}\right): \delta 3.88\left(\mathrm{~s}, 3 \mathrm{H}, \mathrm{OCH}_{3}\right), 5.70(\mathrm{~s}, 1 \mathrm{H}$, $\mathrm{OH}), 6.74-6.78(\mathrm{~d}, J=16.1 \mathrm{~Hz}, 1 \mathrm{H},=\mathrm{CHAr}), 6.89$ (t, 1H, ArH), 6.99-7.01 (d, $J=8.6 \mathrm{~Hz}, 2 \mathrm{H}$, ArH), 7.18 (s, 1H, =CHAr), 7.31-7.33 (d, $J=8.4$ Hz, 2H, ArH), 7.31-7.37( m, 3H, ArH), 7.397.53 ( m, 5H, ArH), 8.11-8.15 (d, $J=16.0 \mathrm{~Hz}, 1 \mathrm{H},=\mathrm{CHAr}), 8.25-8.27$ (d, $J=8.8 \mathrm{~Hz}, 2 \mathrm{H}, \mathrm{ArH})$. ${ }^{13} \mathrm{C} \mathrm{NMR}\left(400 \mathrm{MHz}, \mathrm{CDCl}_{3}\right): \delta 55.39,112.02,113.05,114.50,116.66,119.88,122.52,127.14$, $127.53,128.24,128.49,128.80,129.73,131.48,133.18,134.42,137.74,155.79,158.76,161.64$, 169.66; ESIMS (m/z, \%): $397(\mathrm{M}+1,100)$.

(4Z)-4-(4-Methoxybenzylidene)-2-((E)-3-nitrostyryl)-1-phenyl-1,4-dihydro-5H-imidazolin-

5-one (2e). Orange product; Isolated yield $45 \%$; m.p $165^{\circ}-166^{\circ} \mathrm{C} ; \mathrm{R}_{\mathrm{f}} 0.5\left(80 \% \mathrm{CHCl}_{3}\right.$-Petroleum ether); IR (KBr): 3420, 3071, 2924, 2853, 1711, 1630, 1599, 1532, 1505, 1457, 1389, 1308, 1259, 1166, 1080, $1025 \mathrm{~cm}^{-1} ;{ }^{1} \mathrm{H}$ NMR (400 MHz, $\left.\mathrm{CDCl}_{3}\right): \delta 3.88\left(\mathrm{~s}, 3 \mathrm{H}, \mathrm{OCH}_{3}\right)$, 6.71-6.75 ( $\mathrm{d}, J=15.8 \mathrm{~Hz}, 1 \mathrm{H},=\mathrm{CHAr}), 7.04-7.02(\mathrm{~d}, J=9.0 \mathrm{~Hz}, 2 \mathrm{H}, \mathrm{ArH}), 7.29$ (s, 1H, =CHAr), 7.32-7.35 (d, $J=8.8 \mathrm{~Hz}, 1 \mathrm{H}, \mathrm{ArH}), 7.48-7.59$ ( $\mathrm{m}, 5 \mathrm{H}, \mathrm{ArH}), 7.78-7.80$ ( d, $J=7.8 \mathrm{~Hz}, 1 \mathrm{H}, \mathrm{ArH})$ 8.00-8.04 (d, $J=15.9 \mathrm{~Hz}, 1 \mathrm{H},=\mathrm{CHAr}), 8.18-8.20(\mathrm{dd}, J=8.0 \mathrm{~Hz}, 1 \mathrm{H}, \mathrm{ArH}) 8.27-8.29$ (d, $J=8.9 \mathrm{~Hz}, 2 \mathrm{H}$, $\mathrm{ArH}), 8.31$ (s, $1 \mathrm{H}, \mathrm{ArH}) ;{ }^{13} \mathrm{C} \mathrm{NMR}\left(400 \mathrm{MHz}, \mathrm{CDCl}_{3}\right): \delta 55.43,114.50,116.75,119.88,122.39$, $123.94,124.14,124.44,127.39,128.94,129.47,129.85,133.20,134.69,136.58,136.89,137.39$, 148.66, 156.66, 161.81, 169.66; $\operatorname{ESIMS}(\mathrm{m} / \mathrm{z}, \%): 426(\mathrm{M}+1,100)$. Anal. Calcd for $\mathrm{C}_{25} \mathrm{H}_{19} \mathrm{~N}_{3} \mathrm{O}_{4}: \mathrm{C}$, 70.58; H, 4.50; N, 9.88; O, 15.04. Found: C, 70.51; H, 4.43; N, 9.82; O, 15.24.

(4Z)-4-(4-Methoxybenzylidene)-2-((E)-styryl)-1-phenyl-1,4-dihydro-5H-imidazolin-5-one

(2f). Orange product; Isolated yield 70\%; m.p $143^{\circ}-144^{\circ} \mathrm{C} ; \mathrm{R}_{\mathrm{f}} 0.5\left(80 \% \mathrm{CHCl}_{3}\right.$-Petroleum ether); IR (KBr): 3433, 3069, 2922, 2853, 1707, 1627, 1601, 1501, 1378, 1305, 1261,1166, 1026 $\mathrm{cm}^{-1}$; ${ }^{1} \mathrm{H} \mathrm{NMR}\left(400 \mathrm{MHz}, \mathrm{CDCl}_{3}\right): \delta 3.89\left(\mathrm{~s}, 3 \mathrm{H}, \mathrm{OCH}_{3}\right), 6.59-6.63(\mathrm{~d}, J=15.8 \mathrm{~Hz}, 1 \mathrm{H}$, =CHAr), 7.00-7.02 (d, $J=8.8 \mathrm{~Hz}, 2 \mathrm{H}, \mathrm{ArH}), 7.24$ (s, 1H, =CHAr), 7.34-7.36 (m, 5H, ArH), 7.47-7.57 ( $\mathrm{m}, 5 \mathrm{H}, \mathrm{ArH}), 7.98-8.02(\mathrm{~d}, J=15.8 \mathrm{~Hz}, 1 \mathrm{H},=\mathrm{CHAr}), 8.27-8.29$ (d, $J=8.8 \mathrm{~Hz}, 2 \mathrm{H}$, $\mathrm{ArH}) .{ }^{13} \mathrm{C} \mathrm{NMR}\left(400 \mathrm{MHz}, \mathrm{CDCl}_{3}\right): \delta 55.38,113.66,114.40,127.49,127.53,127.92,128.17$, 
128.67, 129.14, 129.65, 130.05, 133.38, 134.45, 135.16, 136.68, 140.68, 157.59, 161.50, 169.85; ESIMS (m/z, \%): 381(M+ 1, 100). Anal. Calcd for $\mathrm{C}_{25} \mathrm{H}_{20} \mathrm{~N}_{2} \mathrm{O}_{2}$ : C, 78.93; H, 5.30; N, 7.36; O, 8.41. Found: C, 78.85; H, 5.30; N, 7.30; O, 8.55.

\section{Acknowledgements}

RG thanks CSIR and ISRO for financial support. GB thanks CSIR for a Senior Research Fellowship. The Authors also thank RSIC in CDRI Lucknow and IIT Kanpur for spectral data.

\section{References}

1. Daley, R. F.; Daley, S. J. In Organic Chemistry, Daley \& Daley, 2001, Ch. 20, p 1046. (b) Trost, B. M.; Fleming, I.; Heathcock, C. H. In Comprehensive Organic synthesis, Pergamon, Oxford, 1991, Vol 2.

2. Palomo, C.; Oiarbide, M.; Garcia, J. M. Chem. Eur. J. 2002, 8, 36.

3. (a)Casiraghi, G.; Zanardi, F.; Appendino, G.; Rassu, G. Chem. Rev. 2000, 100, 1929. (b) Casiraghi, G.; Zanardi, F.; Rassu, G. Pure Appl. Chem. 2000, 72, 1645.

4. Fringuelli, F.; Pani, G.; Piermatti, O.; Pizzo, F. Tetrahedron 1994, 50, 11499. (b) Ballini, R.; Bosica, G. Tetrahedron. Lett. 1996, 37, 8027. (c) Ballini, R.; Bosica, G. Eur. J. Org. Chem. 1998, 2, 355.

5. Chandrasekhar, S.; Narsihmulu, Ch.; Reddy, N. R.; Sultana, S. S. Chem. Comm. 2004, 2450.

6. Machajewski, T. D.; Wong, Ch.-H. Angew. Chem. Int. Ed. 2000, 39, 1352. (b) Palomo, C.; Oiarbide, M.; Garcia, J. M. Chem. Soc. Rev. 2004, 33, 65.

7. Denmark, S. E.; Stavenger, R. A. Acc. Chem. Res. 2000, 33, 432, and references cited therein. (b) Matsukawa, S.; Okano, N.; Imamoto, T. Tetrahedron Lett. 2000, 41, 103.

8. Groger, H.; Vogel, E. M.; Shibasaki, M. Chem. Eur. J, 4, 1998, 1137. (b) Nelson, S. G.; Tetrahedron Asymmetry 1998, 9, 357. (c) Chan, P. W. H.; Kamijo, S.; Yamamoto, Y.; Synlett 2001, SI, 910.

9. Chiong, W. T.; Wong, M. W. J. Org. Chem. 2005, 70(1), 124.

10. Onitsuka, S.; Nishino, H.; Kurosawa, K. Tetrahedron Lett. 2000, 41, 3149. (b) Corma, S.; Garcia, H.; Chem. Rev. 2003, 103, 4307. (c) Ref 7(c). (d) Periasamy, M. Arkivoc 2002, 7, 151.

11. (a) Chowdari, N. S.; Ramachary, D. B.; Barbas, C. F. III. Synlett 2003, 1906. (b) Cordova, A.; Notz, W.; Barbas, C. F. III. Chem. Commun. 2002, 3024. (c) Darbre, T.; Machuqueiro, M. Chem. Commun. 2003, 1090. (d) Kobayashi, S.; Manabe, K. Acc. Chem. Res. 2002, 35, 209. (e) Ishikawa, S.; Hamada, T.; Manabe, K.; Kobayashi, S. J. Am. Chem. Soc. 2004, 126, 12236. (f) Pandey, R. K.; Dagade, S. P.; Upadhyay, R. K.; Dongare, M. K.; Kumar, P. 
Arkivoc 2002, 7, 28. (g) Liu, G-H; Fan, Q-H; Yang, X-Q; Chen, X-M. Arkivoc 2003, 2 , 123.

12. Pascal, L.; Eyndea, J. J. V.; Haverbekea, Y. V.; Dubois, P. Letters in Organic Chemistry 2004, 112. (b) House, H. O. In Modern Synthetic Reactions, 2nd Edn., Benjamin, W.A, Inc. : Menlo Park, California, 1972.

13. March, J. In Advanced Organic Chemistry, 4th Edn, Wiley, 1992.

14. Tanaka, K.; Toda, F. Chem. Rev. 2000, 100, 1025. (b) Loupy, A. Top. Curr. Chem. 2000, 206, 153. (c) Zahouily, M; Mezdar, A; Elmakssoudi, A; Mounir, B; Rayadh, A; Sebti, S; Lazrek, H. B. Arkivoc 2006, 2, 31.

15. Badr, M. Z.; El-Sherief, H. A. H.; Tadros, M.E. Bull. Chem. Soc. Jpn. 1982, 55, 2267.

16. Bhattacharjya, G.; Savitha, G.; Ramanathan, G. Cryst. Eng. Comm. 2004, 6, 233.

17. Vogel's Textbook of Practical Organic Chemistry, $5^{\text {th }}$ Edn., ELBS Longman Singapore Publishers Pvt. Ltd. 1996.

18. Roncali, J. Annu. Rep. Prog. Chem. Sect. C 1999, 95, 47.

19. Grätzel, M. Journal of Photochemistry and Photobiology C: Photochemistry Reviews 2003, $4,145$. 